\title{
Organizational Factors Affecting Physician Well-Being
}

Daniel S. Tawfik, MD, MS ${ }^{1, *}$

Jochen Profit, MD, $M P H^{2,3}$

Sarah Webber, $M D^{4}$

Tait D. Shanafelt, $M D^{5}$

\author{
Address \\ ${ }^{*}, 1$ Division of Pediatric Critical Care Medicine, Department of Pediatrics, Stanford \\ University School of Medicine, 770 Welch Road, Suite 435, Palo Alto, CA, 94304, \\ USA \\ Email: dtawfik@stanford.edu \\ ${ }^{2}$ Perinatal Epidemiology and Health Outcomes Research Unit, Division of Neona- \\ tology, Department of Pediatrics, Stanford University School of Medicine, \\ Stanford, CA, USA \\ ${ }^{3}$ California Perinatal Quality Care Collaborative, Palo Alto, CA, USA \\ ${ }^{4}$ Department of Pediatrics, University of Wisconsin School of Medicine and Public \\ Health, Madison, WI, USA \\ ${ }^{5}$ Department of Medicine, Stanford University School of Medicine, Stanford, CA, \\ USA
}

Published online: 9 February 2019

(C) Springer Nature Switzerland AG 2019

This article is part of the Topical Collection on Physician Wellness and Burnout

Keywords Organizational factors · Burnout · Physician well-being · Physician engagement

\section{Abstract}

Purpose of review Symptoms of burnout affect approximately half of pediatricians and pediatric subspecialists at any given time, with similarly concerning prevalence of other aspects of physician distress, including fatigue, depressive symptoms, and suicidal ideation. Physician well-being affects quality of care, patient satisfaction, and physician turnover. Organizational factors influence well-being, stressing the need for organizations to address this epidemic.

Recent findings Organizational characteristics, policies, and culture influence physician well-being, and specific strategies may support an environment where physicians thrive. We highlight four organizational opportunities to improve physician well-being: developing leaders, cultivating community and organizational culture, improving practice efficiency, and optimizing administrative policies. Leaders play a key role in aligning organizational and individual values, promoting professional fulfillment, and fostering a 
culture of collegiality and social support among physicians. Reducing documentation burden and improving practice efficiency may help balance job demands and resources. Finally, reforming administrative policies may reduce work-home conflict, support physician's efforts to attend to their own well-being, and normalize use of supportive resources. Summary Physician well-being is critical to organizational success, sustainment of an adequate workforce, and optimal patient outcomes. Because burnout is primarily influenced by organizational factors, organizational interventions are key to promoting wellbeing. Developing supportive leadership, fostering a culture of wellness, optimizing practice efficiency, and improving administrative policies are worthy of organizational action and further research.

\section{Introduction}

The average US physician has a demanding work schedule, experiences intense interpersonal interactions, deals with complex and high-stakes decisions in the face of uncertainty, and must rapidly adapt to new technologies. While many came to the profession inspired by the potential for meaningful work and professional satisfaction, the modern practice environment is burdened by financial stressors, decreasing autonomy, increasing regulatory oversight, decreasing face-to-face time with patients, cultural and structural barriers to self-care, and threats to work-life integration. It is not surprising that physician burnout, a work-related reversible syndrome of emotional exhaustion, depersonalization, and low personal accomplishment, is higher than in the general working population, with symptoms affecting up to half of physicians in active practice [1]. Other dimensions of physician distress include fatigue, stress, depressive symptoms, suicidal ideation, moral distress, and dissatisfaction with work-life integration, many of which have overlapping features with burnout [2].

Nearly half of US pediatricians are experiencing symptoms of professional burnout at any given time according to a 2014 national survey, with general pediatrician burnout significantly more common than just 3 years prior [1]. Less than $50 \%$ of general pediatricians and $40 \%$ of subspecialty pediatricians are satisfied with their work-life integration, highlighting the urgency with which organizational reforms are needed [1]. Although some drivers of burnout may be similar regardless of specialty, pediatricians represent a large and diverse group of physicians with varying practices and patient populations and may experience unique drivers and solutions. For example, pediatrician exposure to the suffering and death of children-a painful and unsettling experience-may lead to significant emotional labor, compassion fatigue, and emotional exhaustion [35]. Furthermore, the complexities of working with families to determine the best interests of a child can be taxing and ethically challenging $[6,7]$.

There is an ethical and moral imperative to address physician burnout, as it contributes to adverse effects at the physician, patient, and organization levels, including physician suicidality, alcohol abuse, broken relationships [8-11], diminished quality of care [12•, 13-15], and impaired retention and recruitment of both physicians and patients [16-23]. Each of these effects threatens the ability of health care organizations to achieve their mission [24]. To go beyond mere elimination of burnout, and achieve the desired outcome of physician well-being, the scope of interventions must be broad and include work and non-work-related issues [25].

Fortunately, although not yet implemented on a wide scale, several effective strategies to address physician burnout and promote well-being have been identified $[26,27]$. Evidence is emerging that personal resilience tools can make a substantial contribution to wellbeing at the individual and work-unit level but need to be undergirded by organizational strategies to further cement their effectiveness and prevent burnout where feasible $[28,29]$. In this review, we summarize specific organizational strategies to improve physician well-being, with an emphasis on four broad categories as shown in Fig. 1: leadership, community and culture, practice efficiency, and administrative policies. 


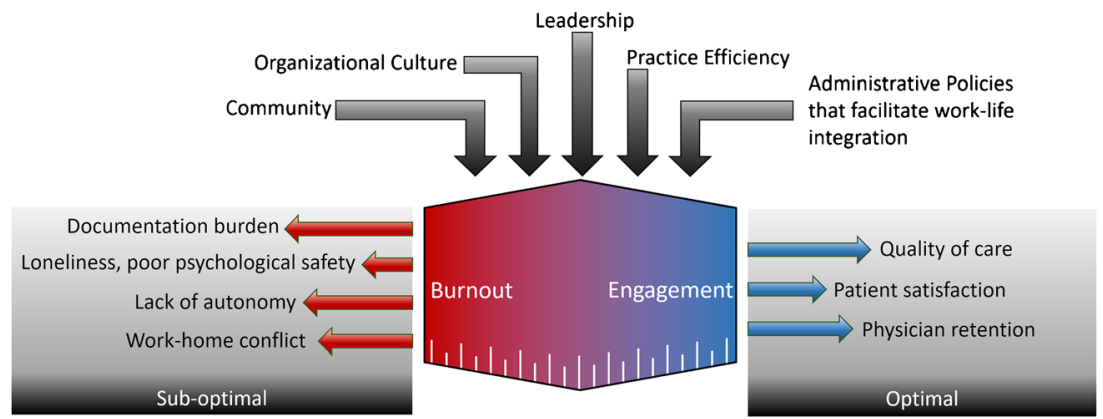

Fig. 1. Key organizational factors affecting physician well-being, and their relation to patient and physician outcomes

\section{Leadership}

\section{Challenges}

The style, perceptions, and quality of organizational leadership can profoundly influence physician well-being. This relationship is increasingly important, as institutions or large practice groups now employ up to $75 \%$ of US physicians [30]. In a recent study of 2800 physicians at the Mayo Clinic, behaviors of the first-line physician leadership explained $11 \%$ of the variance in burnout and $47 \%$ of the variance in job satisfaction among divisions [30]. In fact, each of the 12 leadership qualities evaluated, including those related to transparent communication, humble inquiry, professional development support, and recognition, showed significant relationships with burnout and job satisfaction [30, 31]. Bilal et al. report similar relationships among pediatric nurses, reporting strong associations between burnout and both perceptions of leadership and participation in decision-making [32]. When division chiefs and work-unit leaders function primarily as managers rather than leaders, professional satisfaction and autonomy are decreased [33].

Similarly, job satisfaction strongly relates to how leaders allocate physicians' time. Among academic physicians, Demmy et al. reported a positive correlation between two factors related to time allocation-time for research/personal use and institutional support for clinical/research interests-and job satisfaction [34]. Other studies indicate that physicians who spend greater than $20 \%$ of their time on the aspect of work that is most meaningful to them have a lower likelihood of burnout, suggesting that the identification and promotion of meaningful time allocation may improve physician well-being [35]. In a study of pediatricians, having clinical decision-making autonomy was correlated with less burnout, higher perceived work-life integration, and higher career satisfaction [36]. These findings reflect the long-known association between physician control/autonomy and job satisfaction, further underscored by recent findings of markedly lower burnout prevalence among physicians in small independent practices as compared to the overall US physician population [21,37].

The straightforward solution to addressing these challenges would seem to be selecting and developing physician-leaders with the ability to inform, engage, inspire, and develop those they lead. While it sounds simple, this approach is particularly challenging within the realm of medicine. The training 
and career development invested in creating a high-quality physician is distinctly different and at times antithetical to what is needed to develop a high-quality physician-leader. Physicians often learn to be highly skeptical of new ideas until they are rigorously studied, suppress emotions if they threaten objectivity, and scrutinize minute details in decision-making. These characteristics may empower some physicians to excel scientifically or clinically, but may also pose challenges if these same physicians are thrust into leadership situations that require open consideration of innovative ideas, emotional responsiveness, or big-picture thinking [30].

\section{Opportunities}

With intentionality, the selection and development of high-quality physicianleaders is feasible, and can serve to promote alignment of individual values and organizational priorities-a key construct undergirding physician interactions with leadership [38]. Furthermore, the degree of influence that leadership exerts on burnout and job satisfaction represents a tremendous opportunity for improvement through interventions that promote effective leadership. Strategies include initial selection of leaders based on leadership potential, professional development and directed coaching, and training in leadership skills at all stages of career development [30,31]. These strategies can be further strengthened by dedicated assessment and feedback to leaders, creating accountability for performance in the behaviors that cultivate engagement and development of those they lead [39].

Selecting and training high-quality physician-leaders may require substantial reform for institutions that currently select leaders based on scientific accomplishments or clinical reputation. In the business sector, one study found that selecting leadership based on competencies rather than achievements resulted in the reduction of executive turnover from 25 to $6 \%$ in a large commercial organization, a result that aligns well with the known leadership qualities associated with job satisfaction [40, 41]. Translating these insights to medicine, development of leadership and management skills throughout the career development of physicians is increasingly recognized as an important aspect of medical education, with leadership programs now developed to start at the medical student and resident levels [42-44].

High-quality leadership requires transparent communication and values alignment. Tools for achieving these outcomes can be incorporated into established processes. For example, the annual review between leaders and individual physicians can be optimized by creating a simple conversation template that facilitates clear communication, career development support, and humble inquiry [31]. Organizational strategies that actively solicit physician input regarding work environment and scheduling changes were associated with reduced emotional exhaustion in a study of primary care physicians, and leadership and participation in decision-making were found to promote an improved culture of well-being [45].

Another relatively minor adjustment can take place in the context of leadership WalkRounds $\mathrm{s}^{\mathrm{Tm}}$, a practice by which leaders engage with frontline health care workers in order to identify and resolve issues threatening the safe delivery of care. In a recent evaluation of the WalkRounds ${ }^{\mathrm{TM}}$ practices in 31 hospitals, feedback about improved safety practices was integral to the success of the 
endeavor, suggesting that feedback may improve perceptions of leadership, increase engagement, and support safety practices. More feedback associates with higher engagement among the workforce, but WalkRounds ${ }^{\mathrm{TM}}$ without feedback may have a neutral or negative effect $[46 \bullet, 47,48]$.

\section{Community and organizational culture}

\section{Challenges}

Interpersonal interactions at work can also affect physician well-being. Medicine is a highly interactive profession requiring collegiality, teamwork, shared responsibility for patient safety, and shared experiences of patient outcomes [49]. Safety climate represents a recognition of the shared responsibility for patient safety, and the patterns of behavior intended to minimize patient harm [50]. It is closely related to teamwork climate and is often measured within the same survey instruments, which include the Safety Attitudes Questionnaire and the Hospital Survey on Patient Safety Culture [51-53]. The focus on safety climate of the work-unit is strongly associated with lower rates of burnout among those working in the unit, likely related to its reflection of a commitment to shared values and the resultant impetus for teamwork and collaboration [54].

Teamwork, a core component of patient safety, can mitigate some of the negative effects of excess workload and poor practice efficiency [55]. Beyond simply reducing the workload for individual team members, a strong sense of teamwork can anchor an individual's work experience within a positive social construct that fosters resilience and mutual support [56, 57]. Conversely, poor teamwork can result in increased task load, inefficient workflows, and isolation, which may contribute to emotional exhaustion and depersonalization [54, 58]. In one recent longitudinal study, emotional exhaustion also predicted subsequent poor teamwork, indicating that poor teamwork and burnout may be linked bi-directionally and can create a self-perpetuating cycle [59•].

Social support at work also relates to burnout. Isolation and loneliness among family medicine residents is associated with increased burnout, and a recent study among anesthesiologists found personal support to associate with less emotional exhaustion, suggesting that positive social interactions may mediate burnout in an analogous manner to teamwork interactions [60, 61]. It should also be noted that one study found physicians with emotional exhaustion more likely to seek out personal support, indicating that the relationship between this support mechanism and reduced emotional exhaustion may be even stronger than indicated by cross-sectional studies [62].

\section{Opportunities}

Fostering a community and culture that promotes physician well-being requires concerted effort. Interventions include close leadership involvement in safety and teamwork, facilitating small groups and peer support, and promoting personal resilience.

Leadership WalkRounds ${ }^{\mathrm{TM}}$ and positive rounding — a focus on things that go well-can promote a positive physician culture, improve interactions with leadership (as discussed above), and promote a safety and teamwork climate 


\section{Practice efficiency}

when performed consistently and with appropriate feedback [46•, 47, 63]. A recent cross-sectional analysis of 829 work-units found that units with the most WalkRounds ${ }^{\mathrm{TM}}$ feedback had mean teamwork and safety climate scores that were 10-15 points higher than their counterparts with the least feedback, and burnout scores that were 10 points lower (all on 100-point scales) [46•]. These findings were even more pronounced among neonatal intensive care units, with up to 30-point differences in teamwork and safety climate [63].

Facilitating small groups and other peer support activities may also increase community, decrease isolation, and promote physician well-being if introduced carefully. Successful methods have included physicians in planning small group activities and/or provided physicians choices among different forms of social support $[45,64]$. Two randomized controlled trials in which the organization provided protected time for biweekly physician discussion groups reported decreases in depersonalization as long as 12 months after the intervention, for both facilitated assigned groups and non-facilitated self-selected groups [65, 66]. Organizations can also promote peer support through development of specific programs that provide peer counseling and proactive check-ups for physicians $[67,68]$. As some stigma still may be associated with seeking counseling or support, introducing such programs requires careful thought [68]. Strategies may include attention to the wording of any invitation to peer support, training the peer support volunteers to avoid judgment, and even combining peer support with other forms of physician support such as financial planning services [67]. Finally, organization recognition of the importance of non-work relationships and removal of unnecessary or inefficient work obligations that spill into non-work time (e.g., email, chart completion) can support physicians' non-work relationships.

Organizations can also promote the development of personal resilience skills, and thus contribute to individuals' attention to self-care. These interventions most commonly focus on stress management, mindfulness-based exercises, self-care, or positive psychology, with the majority reporting 4- to 7-point reductions in emotional exhaustion scores (on a 54-point scale) [27].

Supporting these approaches at an organizational level may increase physician engagement while also conveying organizational commitment to well-being. Widespread introduction of these approaches may also promote psychological safety and reduce the stigma often attached to psychological interventions. However, it is likely that physician receptiveness will be greatest when promoting these tools in the context of other organizational change to promote well-being and in combination with protected time or other support. Broad uptake of these practices will likely require that physician and organizational culture place value on self-care and provide time to participate in such activities.

\section{Challenges}

Technological innovation has enabled improved diagnostics and therapeutics, expanded communication with patients and among physicians, and facilitated collection, storage, and retrieval of massive amounts of clinical data [69]. However, these advances have also come at the price of increased clerical burden for physicians. The increase in documentation requirements for billing justification has led to decreased emphasis on the concise and meaningful 
documentation necessary for patient care [70-73]. This evolution in the role of documentation results in massive inefficiencies, with outpatient physicians now spending up to twice as much time on clerical tasks and documentation as they do with patients $[74 \bullet \bullet, 75-76]$. The impact of this shift is increasingly recognized, as physicians with high electronic health record (EHR) task load have higher likelihood of burnout and intent to leave practice, with decreased

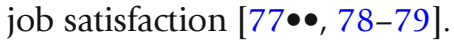

In concert with increasing documentation and regulatory burden, job demands often outweigh job resources. EHR implementation can increase job demands, as physicians spend more time on documentation, order entry, and communication tasks than previously required [80]. If job resources to assist physicians with this burden do not increase concomitantly, EHR implementation can result in a higher likelihood of burnout $[81,82]$. In fact, it is plausible that the increased capital expenditure required for implementation of an EHR could even cause a decrease in job resources through pressure to increase patient volume or reduce staffing, although this relationship has not been proven [83].

Chaos in the work environment is fueled by an imbalance between job demands and resources, and serves as a summary indication of excess patient numbers, complexity, or severity in relation to the resources of the health care system designed to care for them. These resources include the number of health care providers, the individual efficiency of the providers, the level of teamwork, or the overall efficiency of the system. Excess clerical burden, inefficient workflows, and inadequate staffing are among the most prominent contributors to insufficient resources [84]. Chaos is related not only to increased medical errors, but also to physician burnout, stress, and intention to leave practice [84].

\section{Opportunities}

Several strategies exist which may promote practice efficiency and restore the balance between physician job demands and job resources. These strategies range from individual-focused efficiency interventions to expansive reforms of the health care delivery and payment system, and processes in between.

Individual-focused interventions-group or personalized training in EHR efficiency, led by specially trained EHR liaisons or by peers-consistently demonstrate small to moderate improvements in efficiency and satisfaction but do not clearly reduce burnout $[85,86]$. On a slightly larger scale, shifting from a physician-focused to a team-based model of clinical documentation can be particularly effective [87]. The use of scribes (nonlicensed team members specifically trained to document physician-patient encounters) is the best-studied method of reducing physician documentation burden. In one recent study by Gidwani et al., the use of a scribe resulted in a greater than 10-fold increase in the odds of physician satisfaction in a family practice clinic (OR 10.75, 95\% CI 5.3621.58 ) with no change in patient satisfaction [88•]. Similarly, a metaanalysis of eight studies evaluating provider or patient satisfaction with scribes in emergency departments showed consistently positive provider perceptions with unchanged or improved patient satisfaction [89]. Scribes have been shown to be financially feasible, as physicians with scribes are able to see more patients daily and produce documentation with more comprehensive billing codes [90]. 


\section{Administrative policies}

Efficiency improvements at the work-unit level are an effective means for rapidly restoring the balance between job demands and job resources. The most effective interventions are tailored to the specific needs of the clinic or hospital unit, but a few key principles are consistent. First, open collaboration between physicians and organizational leadership may enhance adoption of improvements, by simultaneously addressing physician needs and organizational priorities [33]. Interventions such as pre-visit planning, optimization of patient flow through clinics, and reduction of prior authorization and medication reconciliation tasks all may reduce burnout and improve engagement [45, 91, 92].

Just as excess documentation and regulatory burden can contribute to physician burnout, reduction of that burden may reverse this process. Since 2009, the Health Information Technology for Economic and Clinical Health (HITECH) Act's financial incentives spurred rapid adoption of EHRs across the USA, and in doing so illustrated that federal regulation carries tremendous power to transform health care technology use [72]. Although some of the inefficiencies in EHR use are attributable to specific documentation requirements to report compliance with "meaningful use" guidelines as laid out by HITECH, the potential exists for a modified framework in which requirements decrease the documentation burden [72]. For example, one particularly promising intervention is interoperability-the adoption of communication standards that facilitate seamless sharing of data among health systems and thirdparty developers [69]. Regulation promoting interoperability could thus spur further innovations in automated data extraction for quality benchmarking and research, improved data aggregation and display for clinicians, and more comprehensive patient portals, all while promoting team-based documentation and requiring less input from physicians themselves [69]. Individuals and organizations can support this innovation by advocating for regulatory change coupled to incentives that prioritize interoperability over increased data reporting mandates.

Similarly, the documentation burden imposed by payment within a fee-forservice model will likely require widespread reform for meaningful change [71]. Fee-for-service payment structures mandate highly specific documentation of information that may not have clinical relevance, resulting in unnecessarily long notes that are tedious to write and inefficient to read [73]. Although valuebased payment structures offer hope for reducing the burden of documentation to justify billing, they may counteract this reduction by requiring increased documentation of comorbidities and reporting of quality metrics, highlighting the need for careful consideration when implemented [73].

\section{Challenges}

Although burnout is a work-related phenomenon, the work responsibilities of a physician interact heavily with personal identity, family interactions, and social activities, necessitating a discussion of this interface as a contributor to physician well-being [93]. Work-home conflict is a perceived need to fulfill work and personal responsibilities simultaneously, resulting in a requirement to prioritize one over the other. Such conflicts are highly prevalent (with 44-62\% of physicians and their partners reporting such conflicts in any given 3-week 
period) and are associated with younger age, female sex, academic practice, and higher work hours $[94,95]$. Furthermore, work-home conflict associates with increased burnout and depressive symptoms, lower quality of life, and decreased career satisfaction [94, 96-98].

Administrative policies influence work-home relations on multiple levels, many already discussed above. Leadership style and community can both influence decision-making and priorities when needing to balance demands at home and work, and practice efficiency influences the effective workload [76, 93]. Without safeguards, some payment structures can encourage prioritization of work over family, and physicians paid on an incentive basis report increased work-home conflict and increased burnout [95, 99].

The gender gap in physician well-being may be partially attributed to differences in work-home conflict. Women are more likely to experience both burnout and work-home conflict [17]. Women physician-scientists report that they engage in 8.5 more hours per week of domestic activities than their male counterparts, and they are more than three times as likely to take time off work when usual child care arrangements are disrupted [100]. Women are also nearly three times as likely to believe that child-rearing has hindered their career advancement [95]. These increased rates of work-home conflict among women physicians have been detected as early as 6 months into internship [98]. This issue is particularly important in pediatrics, as $>60 \%$ of the working force is female and among pediatricians, being female is independently associated with lower perceived work-life integration and career satisfaction [36•].

Some of the underlying constructs contributing to these observed demographic differences in well-being are modifiable, with a wide variety of strategies available to address them. For example, allowed parental leave following the birth of a child is extremely variable, with leave ranging from 2 weeks to 1 year in a recent evaluation of top-tier US medical schools [101]. Yet while providing generous support for raising a child or meeting other home commitments is helpful, simply making such options available may not be sufficient. Even in institutions with robust work-life programs, faculty may be unaware of the program, may feel stigmatized for taking advantage of the programs, or may be reluctant to do so because of the effect it would have on their coworkers [102].

Organizations have the opportunity to systematically support the role of physicians as caregivers for children or other loved ones. For women of childbearing age, this may include accommodations for breastfeeding, including time and physical space for pumping [103]. Policies that promote equity among male and female physicians may include flexibility in work schedule to accommodate reduction in work with commensurate pay decreases, providing sick child care, or facilitating clinical coverage for physicians caring for a sick loved one.

Stemming from a recognition that policies alone are insufficient, career-life coaching programs have been developed in which participants work with a coach to create a customized plan identifying and balancing competing priorities, then work directly with their division leadership to implement this plan. Another related strategy is time-banking, which addresses time scarcity by awarding credits to physicians for engaging in team-oriented behaviors (e.g., 


\section{Conclusion}

serving on a selection committee or covering a clinical shift for a colleague on short notice). These credits are then redeemable for personal or professional time-saving services such as home meal delivery or office organizing [104•]. A pilot program that combined career-life coaching and time-banking was recently found to associate with improved wellness and satisfaction, and higher academic achievements than matched controls [104•].

In addition to actively promoting well-being, organizations should also acknowledge the potential for stigmatization and be vigilant against penalizing individuals for acknowledging or seeking support for burnout. Potential penalties include limitations on licensing, hospital privileges, or academic advancement, and may be framed in light of protecting patient safety [9]. Physicians may also fear stigmatization by their peers, particularly in work cultures in which they do not feel safe in asking for help [105]. This phenomenon is best described in the context of physician depression and suicidality, which are distinct from burnout, yet conceptually related [106]. For example, physicians are less likely to seek care for a mental health condition if their state medical licensure application requires them to disclose such conditions [107]. This phenomenon highlights the need for reform of the licensing and privileging system to comply with federal law at a minimum, and to further promote the wellbeing of physicians [106]. Although burnout is a work-related syndrome and not a mental health condition or disability, it may fall prey to a similar phenomenon if this distinction is not recognized.

Organizations must be careful to recognize burnout as an organizational problem indicating a need for process improvement rather than an individual's problem indicating a need for individual action [26]. However, the organization must also promote individual and organizational professionalism by clearly communicating guidelines to develop a culture wherein individuals have the psychological safety to speak up when they need assistance, with those who exhibit unprofessional behavior held accountable [108, 109].

Organizations must also pay careful attention to the authenticity between stated values and financial actions. Organizational leadership has the responsibility to balance fiscal responsibility with the altruistic values of the healing professions. This includes recognition of the fact that productivity-based compensation models may carry the unintended consequences of higher physician burnout, suggesting that appropriate safeguards may be needed to protect against unintended consequences on physician well-being [99, 110].

Physician well-being is critical to organizational success and is primarily driven by organizational factors. Organizational interventions can effectively reduce burnout and promote well-being. Here, we have summarized four organizational strategies to drive progress including: developing physician-leaders, cultivating community and organizational culture, improving practice efficiency, and optimizing administrative policies. In pediatrics, additional emotional burden highlights the urgency of addressing this epidemic. 


\section{Funding}

This work was supported by the Stanford WellMD Center and the Stanford Maternal and Child Health Research Institute. Dr. Profit's effort was in part funded by the Eunice Kennedy Shriver National Institute of Child Health and Human Development [R01 HD084679-01].

\section{Compliance with Ethical Standards}

\section{Conflict of Interest}

Daniel S. Tawfik reports grants from Stanford Maternal and Child Health Research Institute during the conduct of the study. Tait D. Shanafelt reports in addition, Dr. Shanafelt has a patent, Dr. Shanafelt is co-inventor of the Physician Well-being Index, Medical Student Well-Being Index, Nurse Well-being Index, Well-being Index, and the Participatory Management Leadership Index. The Mayo Clinic holds the copyright for this instrument and has licensed it for use outside of Mayo Clinic. Dr. Shanafelt receives a portion of any royalties paid to the Mayo Clinic. As an international expert on the topic of physician well-being, Dr. Shanafelt frequently is asked to present Grand Rounds lectures and keynote presentations as well as provide advising to different healthcare organizations and he frequently receives honoraria for these activities, with royalties paid. Jochen Profit reports grants from NIH, during the conduct of the study. Sarah Webber declares no conflicts of interest.

\section{Human and Animal Rghts and Informed Consent}

This article does not contain any studies with animal subjects performed by any of the authors. All reported studies/ experiments with human subjects performed by the authors have been previously published and complied with all applicable ethical standards (including the Helsinki Declaration and its amendments, institutional/national research committee standards, and international/institutional guidelines).

\section{Publisher's Note}

Springer Nature remains neutral with regard to jurisdictional claims in published maps and institutional affiliations.

\section{References}

Papers of particular interest, published recently, have been highlighted as:

- Of importance

$\bullet \quad$ Of major importance

1. Shanafelt TD, Hasan O, Dyrbye LN, Sinsky C, Satele D, Sloan J, et al. Changes in burnout and satisfaction with work-life balance in physicians and the general US working population between 2011 and 2014. Mayo Clin Proc. 2015;90(12):1600-13.

2. Shanafelt TD, Boone S, Tan L, Dyrbye LN, Sotile W, Satele D, et al. Burnout and satisfaction with work-life balance among US physicians relative to the general US population. Arch Intern Med. 2012;172(18):1377-85.

3. Roth M, Morrone K, Moody K, Kim M, Wang D, Moadel A, et al. Career burnout among pediatric oncologists. Pediatr Blood Cancer. 2011;57(7):1168-73.
4. Shenoi AN, Kalyanaraman M, Pillai A, Raghava PS, Day $\mathrm{S}$. Burnout and psychological distress among pediatric critical care physicians in the United States. Crit Care Med. 2018;46(1):116-22.

5. Boyle DA, Bush NJ. Reflections on the emotional hazards of pediatric oncology nursing: four decades of perspectives and potential. J Pediatr Nurs. 2018;40:6373.

6. Rodriguez-Rey R, et al. Burnout and posttraumatic stress in paediatric critical care personnel: prediction from resilience and coping styles. Aust Crit Care. 2019;32(1):46-53. 
7. Donohue PK, Williams EP, Wright-Sexton L, Boss RD. "It's relentless": Providers' experience of pediatric chronic critical illness. J Palliat Med. 2018;21(7):940-6.

8. Shanafelt TD, et al. Special report: suicidal ideation among American surgeons. Arch Surg. 2011;146(1):54-62.

9. Center C, Davis M, Detre T, Ford DE, Hansbrough W, Hendin $\mathrm{H}$, et al. Confronting depression and suicide in physicians: a consensus statement. JAMA. 2003;289(23):3161-6.

10. Oreskovich MR, Shanafelt T, Dyrbye LN, Tan L, Sotile $\mathrm{W}$, Satele D, et al. The prevalence of substance use disorders in American physicians. Am J Addict. 2015;24(1):30-8.

11. Shanafelt TD, Boone SL, Dyrbye LN, Oreskovich MR, Tan L, West CP, et al. The medical marriage: a national survey of the spouses/partners of US physicians. Mayo Clin Proc. 2013;88(3):216-25.

12. Tawfik DS, et al. Physician burnout, well-being, and work unit safety grades in relationship to reported medical errors. Mayo Clin Proc. 2018;93(11):157180.

This study highlights the relationships among physician burnout, work-unit safety grades, and self-reported errors. Among 6586 physicians in active practice, this study found both burnout and work-unit safety grades to strongly and independently relate to self-reported major medical errors.

13. Shanafelt TD, Balch CM, Bechamps G, Russell T, Dyrbye L, Satele D, et al. Burnout and medical errors among American surgeons. Ann Surg. 2010;251(6):995-1000.

14. West CP, Tan AD, Habermann TM, Sloan JA, Shanafelt TD. Association of resident fatigue and distress with perceived medical errors. JAMA. 2009;302(12):1294300.

15. Poghosyan L, Clarke SP, Finlayson M, Aiken LH. Nurse burnout and quality of care: cross-national investigation in six countries. Res Nurs Health.

2010;33(4):288-98.

16. Shanafelt TD, Mungo M, Schmitgen J, Storz KA, Reeves $\mathrm{D}$, Hayes $\mathrm{SN}$, et al. Longitudinal study evaluating the association between physician burnout and changes in professional work effort. Mayo Clin Proc.

2016;91(4):422-31.

17. Shanafelt TD, Raymond M, Kosty M, Satele D, Horn L, Pippen J, et al. Satisfaction with work-life balance and the career and retirement plans of US oncologists. J Clin Oncol. 2014;32(11):1127-35.

18. Gardiner M, Sexton R, Durbridge M, Garrard K. The role of psychological well-being in retaining rural general practitioners. Aust J Rural Health. 2005;13(3):149-55.

19. Windover AK, Martinez K, Mercer MB, Neuendorf K, Boissy A, Rothberg MB. Correlates and outcomes of physician burnout within a large academic medical center. JAMA Intern Med. 2018;178(6):856-8.

20. Atkinson W, Misra-Hebert A, Stoller JK. The impact on revenue of physician turnover: an assessment model and experience in a large healthcare center. J Med Pract Manage. 2006;21(6):351-5.

21. Buchbinder SB, Wilson M, Melick CF, Powe NR. Primary care physician job satisfaction and turnover. Am J Manag Care. 2001;7(7):701-13.

22. Buchbinder SB, Wilson M, Melick CF, Powe NR. Estimates of costs of primary care physician turnover. Am J Manag Care. 1999;5(11):1431-8.

23. Grunfeld E, Whelan TJ, Zitzelsberger L, Willan AR, Montesanto B, Evans WK. Cancer care workers in Ontario: prevalence of burnout, job stress and job satisfaction. CMAJ. 2000;163(2):166-9.

24. Shanafelt T, Goh J, Sinsky C. The business case for investing in physician well-being. JAMA Intern Med. 2017;177(12):1826-32.

25. Brady KJS, Trockel MT, Khan CT, Raj KS, Murphy ML, Bohman B, et al. What do we mean by physician wellness? A systematic review of its definition and measurement. Acad Psychiatry. 2018;42(1):94-108.

26. Shanafelt TD, Dyrbye LN, West CP. Addressing physician burnout: the way forward. Jama.

2017;317(9):901-2.

27. West CP, Dyrbye LN, Erwin PJ, Shanafelt TD. Interventions to prevent and reduce physician burnout: a systematic review and meta-analysis. Lancet. 2016;388(10057):2272-81.

28. Oliver D. David Oliver: When "resilience" becomes a dirty word. BMJ. 2017;358:j3604.

29. Balme E, Gerada C, Page L. Doctors need to be supported, not trained in resilience. BMJ. 2015;351:h4709.

30. Shanafelt TD, Gorringe G, Menaker R, Storz KA, Reeves D, Buskirk SJ, et al. Impact of organizational leadership on physician burnout and satisfaction. Mayo Clin Proc. 2015;90(4):432-40.

31. Shanafelt T, Swensen S. Leadership and physician burnout: using the annual review to reduce burnout and promote engagement. Am J Med Qual. 2017;32(5):563-5.

32. Bilal A, Ahmed HM. Organizational structure as a determinant of job burnout. Workplace Health Saf. 2017;65(3):118-28.

33. Swensen S, Kabcenell A, Shanafelt T. Physicianorganization collaboration reduces physician burnout and promotes engagement: the Mayo Clinic experience. J Healthc Manag. 2016;61(2):105-27.

34. Demmy TL, Kivlahan C, Stone TT, Teague L, Sapienza $P$. Physicians' perceptions of institutional and leadership factors influencing their job satisfaction at one academic medical center. Acad Med. 2002;77(12 Pt 1):1235-40.

35. Shanafelt TD, West CP, Sloan JA, Novotny PJ, Poland GA, Menaker R, et al. Career fit and burnout among academic faculty. Arch Intern Med. 2009; 169(10):990-5.

36. Starmer AJ, Frintner MP, Freed GL. Work-life balance, burnout, and satisfaction of early career pediatricians. Pediatrics. 2016;137(4):e20153183

This study provides estimates of the career satisfaction and burnout of general and subspecialty pediatricians 9-11 years 
after they completed residency. Among 840 pediatricians, this study found $83 \%$ to be satisfied with their career and $30 \%$ to be experiencing burnout.

37. Blechter B, Jiang N, Cleland C, Berry C, Ogedegbe O, Shelley D. Correlates of burnout in small independent primary care practices in an urban setting. J Am Board Fam Med. 2018;31(4):529-36.

38. Leiter MP, Frank E, Matheson TJ. Demands, values, and burnout: relevance for physicians. Can Fam Physician. 2009;55(12):1224-51225 e1-6.

39. Shanafelt TD, Noseworthy JH. Executive leadership and physician well-being: nine organizational strategies to promote engagement and reduce burnout. Mayo Clin Proc. 2017;92(1):129-46.

40. Lobas JG. Leadership in academic medicine: capabilities and conditions for organizational success. Am J Med. 2006;119(7):617-21.

41. McClelland DC. Identifying competencies with behavioral-event interviews. Psychol Sci. 1998;9(5):331-9.

42. Schwartz RW, Pogge CR, Gillis SA, Holsinger JW. Programs for the development of physician leaders: a curricular process in its infancy. Acad Med. 2000;75(2):133-40.

43. Ackerly DC, Sangvai DG, Udayakumar K, Shah BR, Kalman NS, Cho AH, et al. Training the next generation of physician-executives: an innovative residency pathway in management and leadership. Acad Med. 2011;86(5):575-9.

44. Blumenthal DM, Bernard K, Bohnen J, Bohmer R. Addressing the leadership gap in medicine: residents' need for systematic leadership development training. Acad Med. 2012;87(4):513-22.

45. Dunn PM, Arnetz BB, Christensen JF, Homer L. Meeting the imperative to improve physician well-being: assessment of an innovative program. J Gen Intern Med. 2007;22(11):1544-52.

46. Sexton JB, et al. Providing feedback following Leadership WalkRounds is associated with better patient safety culture, higher employee engagement and lower burnout. BMJ Qual Saf. 2018;27(4):261-7.

This study evaluated the provision of feedback following Leadership WalkRounds ${ }^{\mathrm{TM}}$ in relation to health care provider engagement and burnout. Among 16,797 health care providers from 31 hospitals, this study found WalkRounds ${ }^{\mathrm{TM}}$ with feedback to strongly associate with increased engagement and decreased burnout.

47. Frankel A, Grillo SP, Pittman M, Thomas EJ, Horowitz L, Page $M$, et al. Revealing and resolving patient safety defects: the impact of leadership WalkRounds on frontline caregiver assessments of patient safety. Health Serv Res. 2008;43(6):2050-66.

48. Thomas EJ, Sexton JB, Neilands TB, Frankel A, Helmreich RL. The effect of executive walk rounds on nurse safety climate attitudes: a randomized trial of clinical units[ISRCTN85147255] [corrected]. BMC Health Serv Res. 2005;5(1):28.

49. Van Bogaert P, et al. Predictors of burnout, work engagement and nurse reported job outcomes and quality of care: a mixed method study. BMC Nurs. 2017; 16:5.

50. Singer SJ, Vogus TJ. Safety climate research: taking stock and looking forward. BMJ Qual Saf. 2013;22(1):1-4.

51. Profit J, Etchegaray J, Petersen LA, Sexton JB, Hysong SJ, Mei M, et al. The Safety Attitudes Questionnaire as a tool for benchmarking safety culture in the NICU. Arch Dis Child Fetal Neonatal Ed. 2012;97(2):F127-32.

52. Profit J, Lee HC, Sharek PJ, Kan P, Nisbet CC, Thomas EJ, et al. Comparing NICU teamwork and safety climate across two commonly used survey instruments. BMJ Qual Saf. 2016;25(12):954-61.

53. Sexton JB, Schwartz SP, Chadwick WA, Rehder KJ, Bae J, Bokovoy J, et al. The associations between work-life balance behaviours, teamwork climate and safety climate: cross-sectional survey introducing the work-life climate scale, psychometric properties, benchmarking data and future directions. BMJ Qual Saf. 2017;26(8):632-40.

54. Profit J, Sharek PJ, Amspoker AB, Kowalkowski MA, Nisbet CC, Thomas EJ, et al. Burnout in the NICU setting and its relation to safety culture. BMJ Qual Saf. 2014;23(10):806-13.

55. Smith CD et al. Implementing optimal team-based care to reduce clinician burnout. NAM Perspectives. 2018. https://doi.org/10.31478/201809c.

56. Tawfik DS, Sexton JB, Adair KC, Kaplan HC, Profit J. Context in quality of care: improving teamwork and resilience. Clin Perinatol. 2017;44(3):541-52.

57. Wahlin I, Ek AC, Idvall E. Staff empowerment in intensive care: nurses' and physicians' lived experiences. Intensive Crit Care Nurs. 2010;26(5):262-9.

58. Estryn-Behar M, et al. The impact of social work environment, teamwork characteristics, burnout, and personal factors upon intent to leave among European nurses. Med Care. 2007;45(10):939-50.

59. Welp A, Meier LL, Manser T. The interplay between teamwork, clinicians' emotional exhaustion, and clinician-rated patient safety: a longitudinal study. Critical Care. 2016;20:110-.

This study evaluated the complex interactions among emotional exhaustion, teamwork, and patient safety. Among 2100 health care providers in 55 intensive care units, this study found emotional exhaustion to predict future impaired teamwork, which in turn predicted future impaired patient safety.

60. Hyman SA, Shotwell MS, Michaels DR, Han X, Card EB, Morse JL, et al. A survey evaluating burnout, health status, depression, reported alcohol and substance use, and social support of anesthesiologists. Anesth Analg. 2017;125(6):2009-18.

61. Rogers E, Polonijo AN, Carpiano RM. Getting by with a little help from friends and colleagues: testing how residents' social support networks affect loneliness and burnout. Can Fam Physician. 2016;62(11):e677-83.

62. Bruce SM, Conaglen HM, Conaglen JV. Burnout in physicians: a case for peer-support. Intern Med J. 2005;35(5):272-8.

63. Sexton JB, Sharek PJ, Thomas EJ, Gould JB, Nisbet CC, Amspoker AB, et al. Exposure to Leadership 
WalkRounds in neonatal intensive care units is associated with a better patient safety culture and less caregiver burnout. BMJ Qual Saf. 2014;23(10):814-22.

64. Ro KE, et al. Counselling for burnout in Norwegian doctors: one year cohort study. BMJ. 2008;337:a2004.

65. West CP, Dyrbye LN, Rabatin JT, Call TG, Davidson JH, Multari A, et al. Intervention to promote physician well-being, job satisfaction, and professionalism: a randomized clinical trial. JAMA Intern Med. 2014;174(4):527-33.

66. West $\mathrm{CP}$, et al. A randomized controlled trial evaluating the effect of COMPASS (COlleagues Meeting to Promote and Sustain Satisfaction) small group sessions on physician well-being, meaning, and job satisfaction. J Gen Intern Med. 2015;30:S89.

67. Shanafelt TD, Lightner DJ, Conley CR, Petrou SP, Richardson JW, Schroeder PJ, et al. An organization model to assist individual physicians, scientists, and senior health care administrators with personal and professional needs. Mayo Clin Proc.

2017;92(11):1688-96

68. Shapiro J, Galowitz P. Peer support for clinicians: a programmatic approach. Acad Med.

2016;91(9):1200-4.

69. Washington V, DeSalvo K, Mostashari F, Blumenthal D. The HITECH era and the path forward. N Engl J Med. 2017;377(10):904-6.

70. Martin SA, Sinsky CA. The map is not the territory: medical records and 21 st century practice. Lancet. 2016;388(10055):2053-6.

71. Berenson RA, Basch P, Sussex A. Revisiting E\&M visit guidelines-a missing piece of payment reform. $\mathrm{N}$ Engl J Med. 2011;364(20):1892-5.

72. Halamka JD, Tripathi M. The HITECH era in retrospect. N Engl J Med. 2017;377(10):907-9.

73. Downing NL, Bates DW, Longhurst CA. Physician burnout in the electronic health record era: are we ignoring the real cause? Ann Intern Med. 2018;169(1):50-1.

74.• Sinsky C, et al. Allocation of physician time in ambulatory practice: a time and motion study in 4 specialties. Ann Intern Med. 2016;165(11):753-60

This study evaluated the allocation of outpatient physicians' time. Using 430 hours of observations of 57 physicians; this study found outpatient physicians (family medicine, internal medicine, cardiology, and orthopedics) to spend over twice as much time on desk work as face-to-face contact with patients or colleagues.

75. Tai-Seale M, Olson CW, Li J, Chan AS, Morikawa C, Durbin $\mathrm{M}$, et al. Electronic health record logs indicate that physicians split time evenly between seeing patients and desktop medicine. Health Aff (Millwood). 2017;36(4):655-62.

76. Arndt BG, Beasley JW, Watkinson MD, Temte JL, Tuan WJ, Sinsky CA, et al. Tethered to the EHR: primary care physician workload assessment using EHR event log data and time-motion observations. Ann Fam Med. 2017;15(5):419-26.
77.• Shanafelt TD, et al. Relationship between clerical burden and characteristics of the electronic environment with physician burnout and professional satisfaction. Mayo Clin Proc. 2016;91(7):836-48

This study evaluated the relationship between the use of electronic health records and/or computerized physician order entry and symptoms of burnout. Among 6375 physicians in active practice, this study found overall low satisfaction with electronic health records, and a strong association between computerized physician order entry and burnout despite adjustment for other demographic, clinical, and technological factors.

78. Babbott S, Manwell LB, Brown R, Montague E, Williams E, Schwartz M, et al. Electronic medical records and physician stress in primary care: results from the MEMO Study. J Am Med Inform Assoc. 2014;21(e1):e100-6.

79. Tawfik DS, Phibbs CS, Sexton JB, Kan P, Sharek PJ, Nisbet CC, et al. Factors associated with provider burnout in the NICU. Pediatrics. 2017;139(5):e20164134.

80. Baumann LA, Baker J, Elshaug AG. The impact of electronic health record systems on clinical documentation times: a systematic review. Health Policy. 2018;122(8):827-36.

81. Bakker AB, Demerouti E, Euwema MC. Job resources buffer the impact of job demands on burnout. J Occup Health Psychol. 2005;10(2):170-80.

82. Demerouti E, Bakker AB, Nachreiner F, Schaufeli WB. The job demands-resources model of burnout. J Appl Psychol. 2001;86(3):499-512.

83. Helton J, Langabeer J, DelliFraine J, Hsu C. Do EHR investments lead to lower staffing levels? Healthc Financ Manage. 2012;66(2):54-60.

84. Perez HR, Beyrouty M, Bennett K, Baier Manwell L, Brown RL, Linzer M, et al. Chaos in the clinic: characteristics and consequences of practices perceived as chaotic. J Healthc Qual. 2017;39(1):43-53.

85. Dastagir MT, et al. Advanced proficiency EHR training: effect on physicians' EHR efficiency, EHR satisfaction and job satisfaction. AMIA Annu Symp Proc. 2012;2012:136-43.

86. Stroup K, Sanders B, Bernstein B, Scherzer L, Pachter LM. A new EHR training curriculum and assessment for pediatric residents. Appl Clin Inform. 2017;8(4):9941002.

87. Sinsky CA, Willard-Grace R, Schutzbank AM, Sinsky TA, Margolius D, Bodenheimer T. In search of joy in practice: a report of 23 high-functioning primary care practices. Ann Fam Med. 2013;11(3):272-8.

88. Gidwani R, et al. Impact of scribes on physician satisfaction, patient satisfaction, and charting efficiency: a randomized controlled trial. Ann Fam Med. 2017;15(5):427-33

This randomized crossover trial evaluated the effect of introduction of scribes on physician/patient satisfaction and charting efficiency in a small family practice. The four physicians in the study reported improved satisfaction with clinic 
and charting accuracy for weeks with scribes present, with no change in patient satisfaction noted.

89. Heaton HA, Castaneda-Guarderas A, Trotter ER, Erwin PJ, Bellolio MF. Effect of scribes on patient throughput, revenue, and patient and provider satisfaction: a systematic review and meta-analysis. Am J Emerg Med. 2016;34(10):2018-28.

90. Bank AJ, Gage RM. Annual impact of scribes on physician productivity and revenue in a cardiology clinic. Clinicoecon Outcomes Res. 2015;7:489-95.

91. Rao SK, Kimball AB, Lehrhoff SR, Hidrue MK, Colton DG, Ferris TG, et al. The impact of administrative burden on academic physicians: results of a hospital-wide physician survey. Acad Med. 2017;92(2):237-43.

92. Linzer M, Poplau S, Grossman E, Varkey A, Yale S, Williams E, et al. A cluster randomized trial of interventions to improve work conditions and clinician burnout in primary care: results from the Healthy Work Place (HWP) Study. J Gen Intern Med. 2015;30(8):1105-11.

93. Geurts S, Rutte C, Peeters M. Antecedents and consequences of work-home interference among medical residents. Soc Sci Med. 1999;48(9):1135-48.

94. Dyrbye LN, Sotile W, Boone S, West CP, Tan L, Satele D, et al. A survey of U.S. physicians and their partners regarding the impact of work-home conflict. J Gen Intern Med. 2014;29(1):155-61.

95. Dyrbye LN, Shanafelt TD, Balch CM, Satele D, Sloan J, Freischlag J. Relationship between work-home conflicts and burnout among American surgeons: a comparison by sex. Arch Surg. 2011;146(2):211-7.

96. Dyrbye LN, West CP, Satele D, Sloan JA, Shanafelt TD. Work/home conflict and burnout among academic internal medicine physicians. Arch Intern Med. 2011;171(13):1207-9.

97. Dyrbye LN, et al. Work-home conflicts have a substantial impact on career decisions that affect the adequacy of the surgical workforce. Arch Surg. 2012;147(10):933-9.

98. Guille C, Frank E, Zhao Z, Kalmbach DA, Nietert PJ, Mata DA, et al. Work-family conflict and the sex difference in depression among training physicians. JAMA Intern Med. 2017;177(12):1766-72.

99. Shanafelt TD, Balch CM, Bechamps GJ, Russell T, Dyrbye L, Satele D, et al. Burnout and career satisfaction among American surgeons. Ann Surg. 2009;250(3):463-71.

100. Jolly S, Griffith KA, DeCastro R, Stewart A, Ubel P, Jagsi R. Gender differences in time spent on parenting and domestic responsibilities by high-achieving young physician-researchers. Ann Intern Med. 2014;160(5):344-53.

101. Riano NS, Linos E, Accurso EC, Sung D, Linos E, Simard JF, et al. Paid family and childbearing leave policies at top US medical schools. JAMA. 2018;319(6):611-4.

102. Shauman K, Howell LP, Paterniti DA, Beckett LA, Villablanca AC. Barriers to career flexibility in academic medicine: a qualitative analysis of reasons for the underutilization of family-friendly policies, and implications for institutional change and department chair leadership. Acad Med. 2018;93(2):246-55.

103. Grinberg C. Pumped. JAMA. 2018;320(10):977-8.

104. Fassiotto M, Simard C, Sandborg C, Valantine H, Raymond J. An integrated career coaching and timebanking system promoting flexibility, wellness, and success: a pilot program at Stanford University School of Medicine. Acad Med. 2018;93(6):881-7

This study reports the outcomes from a pilot program of careerlife planning and time-banking in a large academic medical center. Among 60 faculty participating in the program, this study found improved institutional satisfaction and wellness compared to matched controls, in addition to increased academic productivity.

105. Swendiman RA, Edmondson AC, and Mahmoud NN. Burnout in surgery viewed through the lens of psychological safety. Ann Surg. 2019;269(2):234-5.

106. Schroeder R, Brazeau CMLR, Zackin F, Rovi S, Dickey J, Johnson MS, et al. Do state medical board applications violate the Americans with disabilities act? Acad Med. 2009;84(6):776-81.

107. Dyrbye LN, West CP, Sinsky CA, Goeders LE, Satele DV, Shanafelt TD. Medical licensure questions and physician reluctance to seek care for mental health conditions. Mayo Clin Proc. 2017;92(10):1486-93.

108. Egener B, McDonald W, Rosof B, Gullen D. Perspective: organizational professionalism: relevant competencies and behaviors. Acad Med. 2012;87(5):66874 .

109. Egener BE, Mason DJ, McDonald WJ, Okun S, Gaines $\mathrm{ME}$, Fleming DA, et al. The charter on professionalism for health care organizations. Acad Med. 2017;92(8):1091-9.

110. Shanafelt TD, Gradishar WJ, Kosty M, Satele D, Chew $\mathrm{H}$, Horn $\mathrm{L}$, et al. Burnout and career satisfaction among US oncologists. J Clin Oncol. 2014;32(7):678-86. 